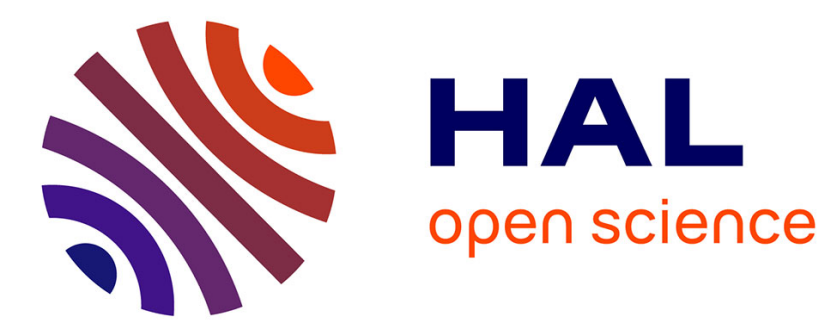

\title{
Guard Interval Adaptation for In-home Power Line Communication
}

Thanh Nhân Vo, Karine Amis Cavalec, Thierry Chonavel, Pierre Siohan

\section{To cite this version:}

Thanh Nhân Vo, Karine Amis Cavalec, Thierry Chonavel, Pierre Siohan. Guard Interval Adaptation for In-home Power Line Communication. ISPLC 2015: 19th International symposium of Power Line Communications and Its Applications, Mar 2015, Austin, Texas, United States. pp.245 - 249, 10.1109/ISPLC.2015.7147622 . hal-01197420

\section{HAL Id: hal-01197420 \\ https://hal.science/hal-01197420}

Submitted on 11 Sep 2015

HAL is a multi-disciplinary open access archive for the deposit and dissemination of scientific research documents, whether they are published or not. The documents may come from teaching and research institutions in France or abroad, or from public or private research centers.
L'archive ouverte pluridisciplinaire HAL, est destinée au dépôt et à la diffusion de documents scientifiques de niveau recherche, publiés ou non, émanant des établissements d'enseignement et de recherche français ou étrangers, des laboratoires publics ou privés. 


\title{
Guard Interval Adaptation for In-home Power Line Communication
}

\author{
Thanh Nhan $\mathrm{VO}^{1}$, Karine AMIS ${ }^{1}$, Thierry CHONAVEL ${ }^{1}$, Pierre SIOHAN ${ }^{2}$ \\ 1: Telecom Bretagne / UMR CNRS 6285 Lab-STICC; 2: Orange Labs
}

\begin{abstract}
This paper aims to analyze the choice of the guard interval (GI) length in PLC systems to optimize the achievable throughput under power and symbol error-rate (SER) constraints. In general, the GI length is chosen so that there is no interference, i.e. the GI length is greater than or equal to the channel impulse response length. However, many previous works have shown that in PLC systems, this GI choice is inefficient in terms of achievable throughput. Indeed, shorter GI evidently results in inter-symbol interference (ISI) and intercarrier interference (ICI), but the gain offered by shortened GI may exceed the loss caused by interference. In this paper, we propose a simple solution for the GI length adaptation in PLC systems to optimize the achievable throughput.
\end{abstract}

Index Terms-Power Line Communication, Guard Interval Adaptation, Throughput maximization, Bit-loading, WindowedOFDM.

\section{INTRODUCTION}

Recently, in-home power line communication (PLC) has received a lot of attention from both the industry and research community. Thanks to its simple installation that does not demand further infrastructures, PLC has been considered as an efficient medium to distribute multimedia contents for inhome networking. To avoid interference with many radio applications that already exist in the band from $0-30 \mathrm{MHz}$ such as amateur radio, urgency and military services, a spectral mask has been specified for PLC systems [1], [2]. To adapt to the spectral mask, the IEEE P1901 standard uses the WindowedOFDM instead of the conventional OFDM technique [2].

In many previous works such as [3], [4], [5], [6], [7], it has been shown that the choice of a GI length equal to the channel impulse response length is inefficient in terms of system throughput. When we insert a GI length equal to $\mu$, the transmission rate is reduced by a factor of $\frac{T_{0}}{T_{0}+\mu}$ where $T_{0}$ is the OFDM symbol length. The bit-loading and statistical GI adaptation for PLC systems has been studied in [4], [5]. However, this allocation is based on the conventional OFDM while, as mentioned above, PLC systems exploit the Windowed-OFDM instead of the conventional OFDM. Moreover, the power allocation in [4], [5] is sub-optimal. Actually, the discretized bit-loading can be achieved with lower power consumption compared to the approach in [4] and the residual power could be exploited to increase data rate or/and transmission quality. GI design for maximizing the achievable throughput for Windowed-OFDM systems is considered in [6]. Unfortunately, the power allocation strategy in [6] is also sub-optimal. Recently, another work for joint GI adaptation and power loading based on the interference calculation for the conventional OFDM systems has been reported in [7]. Several approaches to search for the upperbound of the achievable throughput have been studied in [7] but no practical solution of the achievable throughput maximization problem with low-complexity has been given. In this paper, we use the reduced complexity algorithm proposed in [8] to optimize the achievable throughput for a given GI length and compare the achieved throughput for different GI lengths to choose the optimal GI length. Moreover, based on the simulation results, we propose a simple way to determine the optimal GI length.

The organization of the paper is as follows. In Section II, the throughput maximization problem in Windowed-OFDM based systems is shown and a conventional solution is discussed. In Section III, we propose a simple algorithm to choose the GI value that maximizes the achievable throughput. Simulation results are reported in Section IV. The conclusions are given in Section V.

\section{ACHIEVABLE THROUGHPUT OPTIMIZATION PROBLEM}

In this work, we consider a Windowed-OFDM system with $L$ used subcarriers out of $M$, a roll-off interval of the filter at receiver $R I$ and a guard interval $G I$ defined as in [8]. Let $\mathcal{A}$ be the set of allowable number of bits associated to allowable modulations specified by the standard and $\mathcal{T}_{d}$ be the down discretization function defined as

$$
\mathcal{T}_{d}\left(\mathcal{R}^{+} \rightarrow \mathcal{A}\right): x \rightarrow \max \{T \in \mathcal{A}: \quad T \leq x\}
$$

The problem of bit, power and guard interval adaptation to optimize the throughput is to find $G I=\mu$ and power allocation vector $\mathbf{P}$ so that

$$
\left\{\begin{array}{l}
\max _{\mu, \mathbf{P}} \frac{T_{0}}{T_{0}+\mu} \sum_{m=1}^{L} \mathcal{T}_{d}\left(\log _{2}\left(1+\frac{P(m)|\alpha(m)|^{2}}{\left(\left[\mathbf{W}^{(\mu)} \mathbf{P}\right](m)+N(m)\right) \Gamma}\right)\right) \\
\sum_{m=1}^{L} P(m) \leq P_{\text {total }} \\
0 \leq P(m) \leq P_{\text {max }}(m), \quad \forall m \in[1, L]
\end{array}\right.
$$

where $\alpha(m), P(m),\left[\mathbf{W}^{(\mu)} \mathbf{P}\right](m), N(m)$ are the channel gain, the power allocated, the interference power (due to the central limit theorem, it can be considered as a Gaussian noise [9], [10], [11]) and the noise power on active subcarrier $m$. $\mathbf{W}^{(\mu)}$ is the interference power coefficient matrix and $\Gamma$ is the SNR gap [12]. For further details about $\mathbf{W}^{(\mu)}$ calculation, interested readers can refer to [8].

Indeed, problem (2) can be solved by using the conventional Greedy algorithm, which is a popular tool for discrete optimization problems [13], to evaluate the achievable throughput 
for different $G I$ values and choose the optimal one. We recall that in the conventional Greedy algorithm, to optimize the achievable throughput given a $G I$ value, at every iteration and for each subcarrier, we have to calculate the power required to load up one bit. The subcarrier associated to the minimum required power and such that all power constraints are satisfied is chosen to be loaded up. In [8], it is shown that the complexity to calculate the power after adding one bit on a subcarrier is equivalent to an inversion of a matrix of size $L x L$. Thus, the total complexity for one iteration in the conventional Greedy algorithm is the product of the number of subcarriers that can be loaded up and the complexity of a matrix inversion. Due to its important complexity, the conventional Greedy algorithm can not be used in practical systems.

To reduce the computational cost, in [8], we have proposed the Reduced Complexity Algorithm (RCA). Its principle consists in the simplification of the cost function (which is used to determine the subcarriers to be loaded up) in the Greedy procedure, the use of a judicious initial bit vector different from the null one used in the conventional Greedy approach and the efficient selection of $K$ subcarriers to load up at every iteration. The main advantage of the RCA is that it can converge to almost the same achievable throughput as the one obtained by the conventional Greedy algorithm while it significantly outperforms the conventional Greedy algorithm in terms of complexity. Unfortunately, even when RCA is used instead of the conventional Greedy algorithm, the complexity of a brute-force search for the optimal GI value remains high. To further reduce the complexity, we propose here two methods to search for the optimal GI value.

\section{A. Comparison of the simplified brute-force search and the iterative branch-and-bound search to determine $\mu_{o p t}$}

Let us denote by $\tau_{\max }$ the maximum delay of in-home PLC channels that is about $5.56 \mu s$ [4]. Note that the optimal GI search interval in PLC systems is $\left[R I, \tau_{\max }+R I\right]$. The first method deals with a simplified brute-force (SBF) search, i.e. we only take into account the discretized GI values $\mu=R I+l \Delta(\Delta=0.2 \mu \mathrm{s})$ and $\mu \leq \tau_{\max }+R I$. We have demonstrated that the throughput obtained with this method is about the same as the optimal one obtained with the full bruteforce search, i.e. the throughput degradation is less than $0.5 \%$. However, for the sake of paper length, we do not show the proof here. Second, we exploit the iterative branch-and-bound $(\mathrm{BnB})$ search proposed in [7] for the interval $\left[R I, \tau_{\max }+R I\right]$, i.e. at every iteration, we try to exclude the values of $\mu$ such that the upper bound of their achievable throughput is less than or equal to the best achievable throughput value found so far. The throughput and complexity comparisons between the SBF and the BnB are illustrated in Fig. 1 and 2. For the sake of visibility, we only show the simulation results for 20 channel realizations out of 250 . We can see that the throughput obtained by the SBF search is the same as the one achieved by the $\mathrm{BnB}$. However, the $\mathrm{BnB}$ search significantly reduces the complexity w.r.t the SBF search. The average achievable throughput obtained by the $\mathrm{BnB}$ and $\mathrm{SBF}$ are 237 and 236 (Mbits), respectively. The average number of iterations to

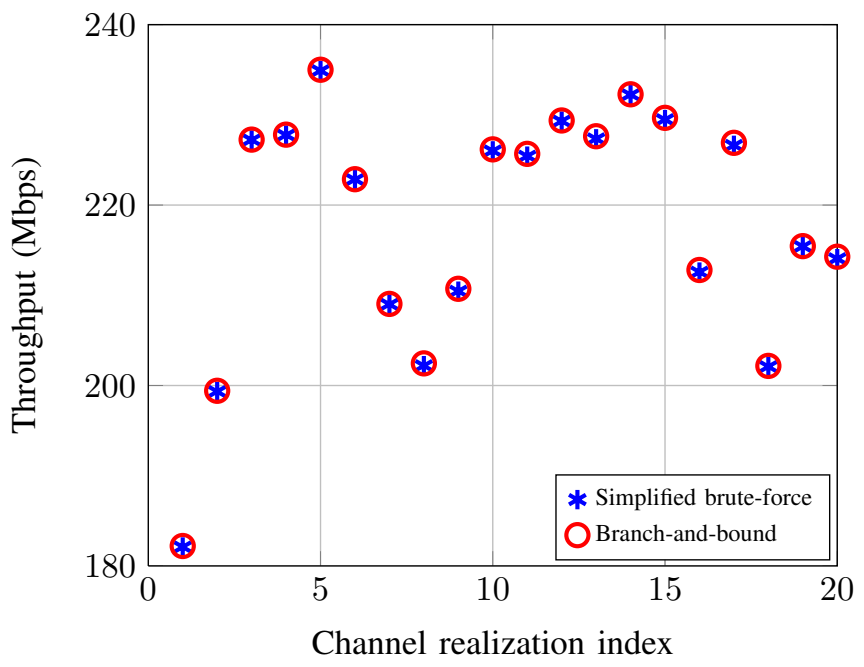

Fig. 1: Throughput comparison between SBF and BnB search.

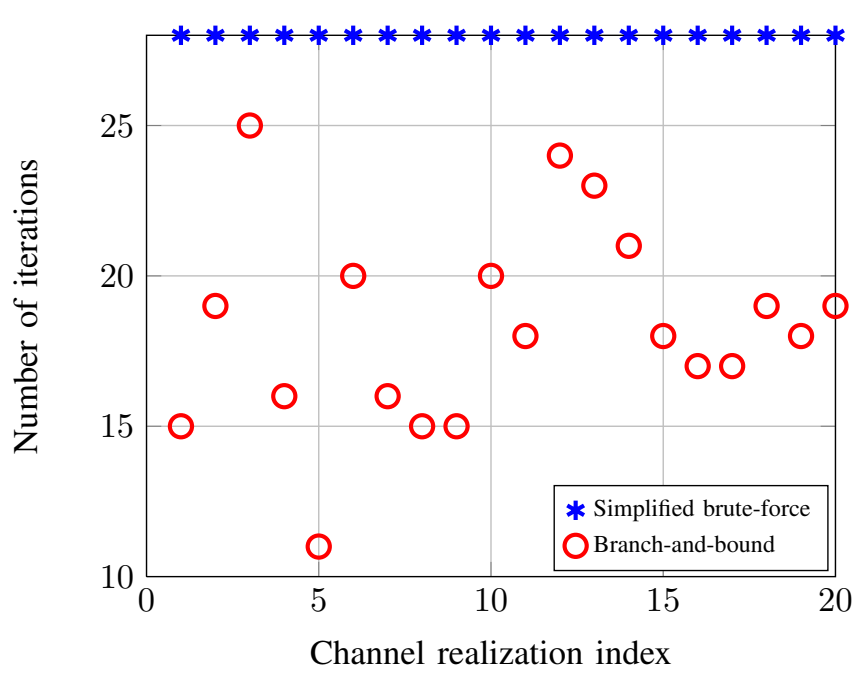

Fig. 2: Comparison of the iteration number between SBF and $\mathrm{BnB}$ search.

determine the optimal GI value is 18 for $\mathrm{BnB}$ and 28 for $\mathrm{SBF}$ and the average run-time per iteration is about 130 seconds for $\mathrm{BnB}$ and 110 seconds for $\mathrm{SBF}$.

\section{B. Linear regression to calculate GI length}

In the literature, many works have claimed that it is better to let the small taps 'escape' the GI [14], [15]. In other words, when the tail of CIR contains only little energy, we can choose a GI shorter than the length of CIR to improve the achievable throughput. In this paper, we evidence and exploit a statistical linear relation between $\mu_{\text {opt }}$ and $\tau_{95}+R I$, where $\tau_{95}$ denote the interval that contains $95 \%$ of power of the CIR. We compared in Fig. $3 \mu_{\text {opt }}$ and $\tau_{95}+R I$ obtained by the iterative branchand-bound search.

We observe that $\mu_{o p t}<\tau_{95}+R I$ and that both evolutions are quite identical. Hence, we propose two models to fix the value of $\mu$ based on $\tau_{95}$ : the first model imposes $\mu=\tau_{95}+R I$ and the second one considers the linear approximation $\mu=$ 


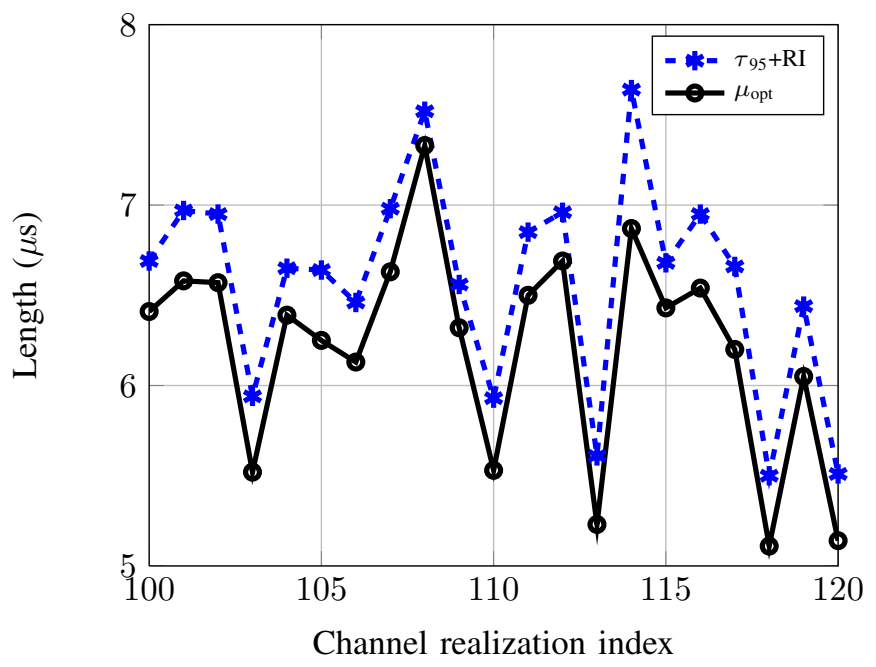

Fig. 3: $\mu_{\text {opt }}$ and $\tau_{95}+R I$.

$\alpha\left(\tau_{95}+R I\right)+\beta$. By using a linear regression, we have obtained $\alpha \approx 1$ and $\beta \approx-0.33$. The GI value obtained with both models and the optimum GI value are shown in Fig. 4.

\section{Simulation RESUlts}

The simulation parameters are given as follows:

- SISO-PLC system with the IEEE P1901 standard.

- $T_{0}=40.96(\mu s), R I=4.96(\mu s)$.

- $\mathcal{A}=\{0,1,2,3,4,6,8,10,12\}$.

- $P_{\text {max }}(m)=1$ (normalized to the spectral mask at $\left.P_{\text {mask }}=-50 \mathrm{dBm} / \mathrm{Hz}\right), \forall m \in[1, L]$. In this paper, we only take into account the spectral mask constraint $P(m) \leq P_{\max }(m)$ that is usually used in PLC systems. It means that the total power constraint is not considered in this work.

- $\Gamma=4.038$, corresponding to SER $=10^{-3}$.

- Channel impulse response $h(t)$ is obtained by the Inverse Fast Fourier Transform (IFFT) of the frequency response of Tonello model and truncated at $\tau_{\max }=5.56 \mu \mathrm{s}$ [16].

- Noise model: Background noise (Esmailian model) [17].

- Number of channel realizations: 250.

In this section, a performance comparison between the following approaches is shown:

- Proposed approach: use the one of two models of linear regression $\mu=\tau_{95}+R I$ (model 1) and $\mu=\alpha\left(\tau_{95}+\right.$ $R I)+\beta$ (model 2) where $\alpha=1$ and $\beta=-0.33$.

- Conventional approach: $\mu=\tau_{\max }+R I$ to ensure entire interference absorption.

- PLC standard approach: use a GI value in a predefined GI set to fully eliminate interference. The GI set used in the IEEE P1901 standard is $[5.56,7.56,9.56,11.56,15.56,19.56,47.12] \mu \mathrm{s}$.

For the visibility, we only illustrate in the figures the simulation results for 20 channel realizations out of 250 . Figures 5 and 6 show the GI values obtained with different approaches and the corresponding achievable throughput, respectively. We can see that our proposed approach (both proposed models)

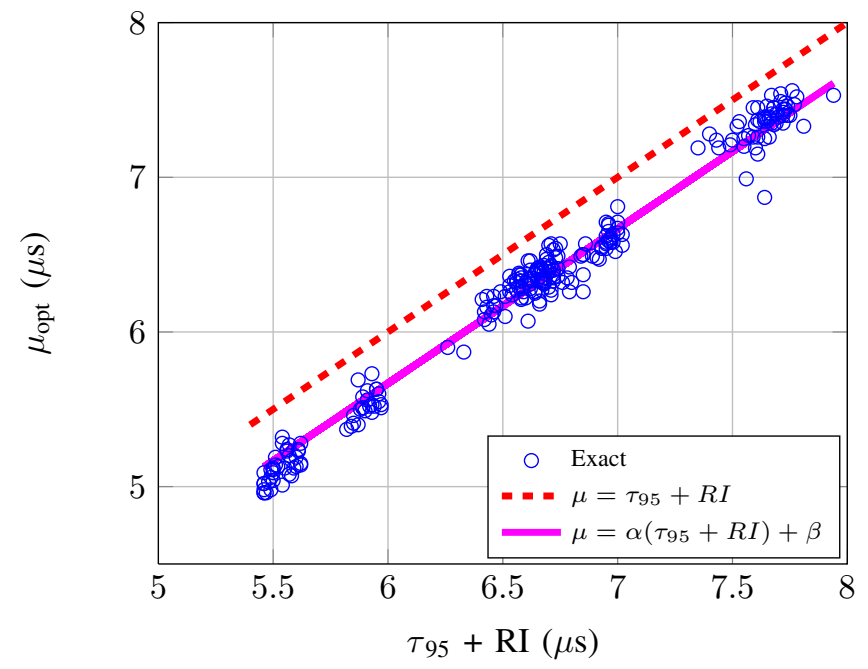

Fig. 4: Linear regression between $\mu_{\text {opt }}$ and $\tau_{95}+R I$.

\begin{tabular}{|l|c|c|c|}
\hline Approach & $\min$ & $\max$ & average \\
\hline Conventional & 139.10 & 211.35 & 202.97 \\
\hline Standard & 136.34 & 207.17 & 198.95 \\
\hline SBF & 148.15 & 235.92 & 220.85 \\
\hline BnB & 148.41 & 236.94 & 221.03 \\
\hline Model 1 & 147.34 & 234.39 & 219.71 \\
\hline Model 2 & 148.26 & 236.10 & 220.74 \\
\hline
\end{tabular}

TABLE I: Minimal, maximal and average throughput obtained with different approaches (Mbits).

always chooses more judiciously the GI value than the conventional approach and the approach used in the IEEE P1901 standard. Thus, the achievable throughput obtained by our proposed approach is always higher than the ones achieved by the conventional and IEEE P1901 standard approaches. In addition, this achievable throughput is almost the same w.r.t the optimal one, obtained by the iterative branch-and-bound search or by the simplified brute-force search. However, the complexity (expressed through the number of iterations) of our proposed algorithm is significantly reduced because we don't need any search. Note that in the SBF as well as the $\mathrm{BnB}$ search procedures, for one iteration (with a given GI), we have to calculate all entries of the interference power coefficient matrix $\mathbf{W}$ and exploit the RCA in [8] to optimize the achievable throughput corresponding to this GI value. Thus, since our proposed approach doesn't need any search procedure, thus it will strongly reduce the computation cost of the GI adaptation.

The maximal, minimal and average values of the throughput obtained with different approaches are given in Table I. We observe that in all cases, both proposed models outperform by $10 \%$ the conventional and standard approaches. In addition, when compared to the iterative branch-and-bound or simplified brute-force search, the throughput degradation is negligeable ( $0.6 \%$ for the first model and $0.3 \%$ for the second one). As mentioned above, both proposed models significantly reduce the number of iterations, i.e. no iteration versus 18 iterations (in average) for the $\mathrm{BnB}$ search and versus 28 iterations for 


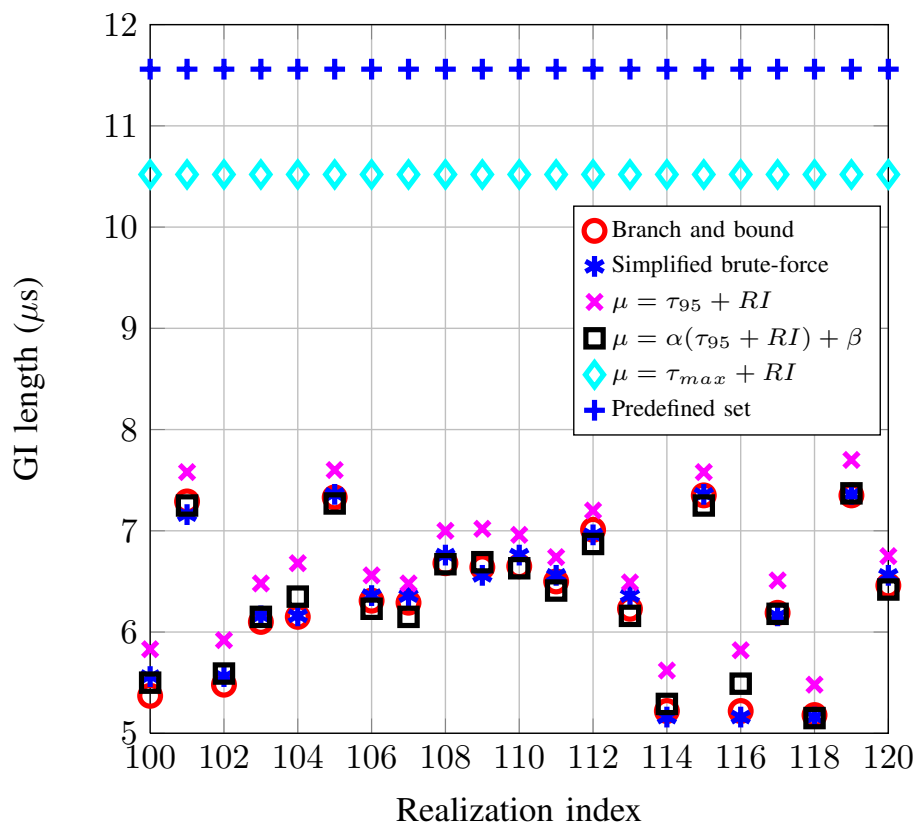

Fig. 5: GI adaptation with different approaches.

SBF search. This confirms that both proposed models can be used to calculate the quasi-optimal GI value.

Finally, the joint optimization problem of GI adaptation and bit-loading in PLC systems can be solved by using the concatenation of the proposed GI models and the RCA algorithm. It means that first a GI value can be found with the proposed models and then the RCA algorithm is used to derive the optimal bit/power allocation. The complexity of this concatenation is mainly due to the RCA algorithm. However, let us recall that its complexity is significantly reduced as compared to the conventional greedy algorithm [8].

\section{CONCLUSION AND PERSPECTIVE}

In this work, we have proposed two statistical models to adapt the GI length to optimize the achievable throughput in Windowed-OFDM systems. Both models rely on a statistical relation between the optimal GI value and $\tau_{95}$, i.e the interval that contains $95 \%$ of the power of the channel impulse response. The efficiency of the proposed models has been verified through simulation results in the context of PLC systems, i.e. its complexity is significantly reduced with negligeable degradation in terms of achievable throughput as compared to many approaches such as brute-force search and branch-and-bound search. Moreover, a combination of the proposed models and the greedy based reduced complexity algorithm proposed in [8] can be used to solve the joint optimization problem of GI adaptation and bit/power allocation in Windowed-OFDM systems. The future work will aim to find a set of predefined GI values based on proposed statistical models to reduce the overhead due to the feedback information and test the proposed approach with practical channel estimation.

\section{REFERENCES}

[1] H. P. Alliance, HomePlug AV baseline specification., 2007.

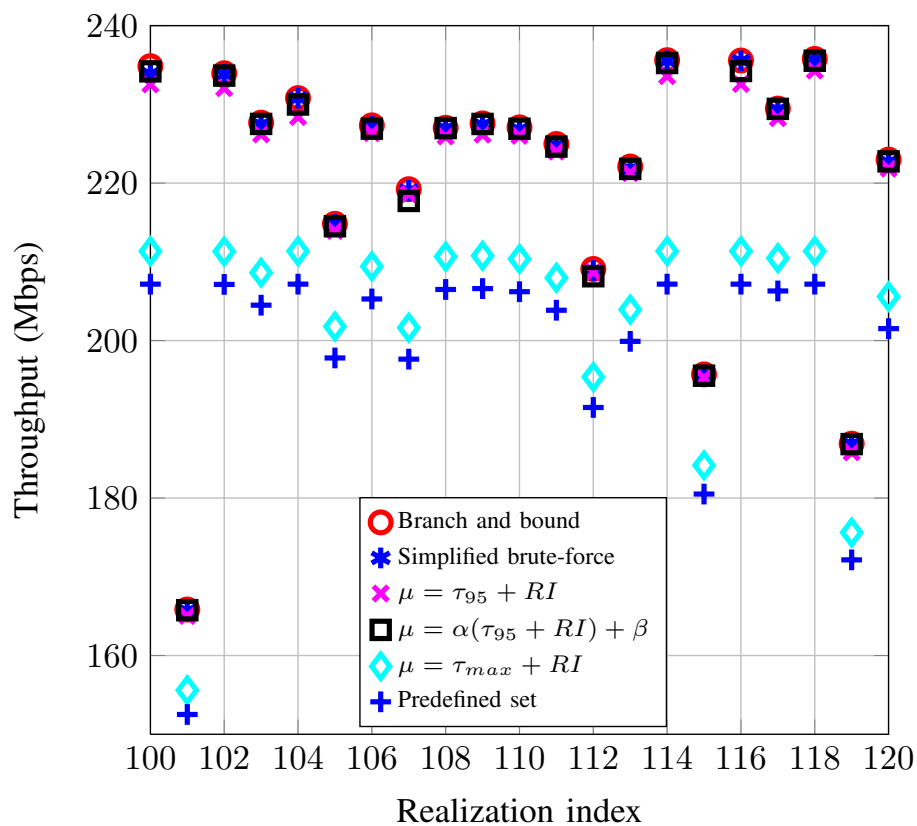

Fig. 6: Achievable throughput with different approaches.

[2] I. C. Society, IEEE Standard for Broadband over Power Line Networks: Medium Access Control and Physical Layer Specification., 2010.

[3] S. Galli, H. Koga, and N. Kodama, "Advanced Signal Processing for PLCs: Wavelet-OFDM.” ISPLC 2008, pp. 187-192, 2008.

[4] M. Tonello, S. Alessandro, and L. Lampe, "Cyclic prefix design and allocation in bit-loaded OFDM over plc channels." IEEE Transactions on Communications, vol. 58(11), pp. 3265-3276, 2010.

[5] M. Ouzzif and J. L. Masson, "Statistical analysis of the cyclic prefix impact on indoor PLC capacity," IEEE ISPLC, pp. 285-289, 2009.

[6] D. Alessandro, M. Tonello, and L. Lampe, "Adaptive pulse-shaped OFDM with application to in-home power line communication," Telecommunication systems, vol. 51(1), pp. 3-13, 2012.

[7] M. Wolkerstorfer, P. Tsiaflakis, M. Moonen, and D. Statovci, "Joint power-loading and cyclic prefix length optimization for OFDM-based communication." IEEE ICASSP, 2013.

[8] T. N. Vo, K. Amis, T. Chonavel, and P. Siohan, "Achievable Throughput Optimization in OFDM Systems in the Presence of Interference and its Application to Power Line Networks," IEEE Transactions on Communications, vol. 62(5), pp. 1704-1715, 2014.

[9] E. Viterbo and K. Fazel, "How to combat long echoes in OFDM transmission scheme: Sub-channel equalization or more powerful channel coding." IEEE GLOBECOM, pp. 2069-2074, 1995.

[10] J. L. Seoane, S. K. Wilson, and S. Gelfand, "Analysis of Intertone and Interblock Interference in OFDM when the length of the Cyclic Prefix is shorter than the length of the Impulse Response of the channel," IEEE GLOBECOM, pp. 32-36, 1997.

[11] A. Cortés, J. Cañete, L. Díez, and M. Torres, "On PLC channel models: an OFDM-based comparison," IEEE 17th ISPLC, pp. 333-338, 2013.

[12] J. M. Cioffi, A multicarrier prime., http://www.stanford.edu/group/cioffi/ publications.html.

[13] H. Cormen, E. Leiserson, L. Rivest, and C. Stein, Introduction to Algorithms. MIT Press, 2001.

[14] W. Henkel, G. Taubock, P. Odling, P. Borjesson, N. Petersson, and 
A. Johansson, "The Cyclic Prefix of OFDM/DMT An Analysis," International Zurich Seminar on Broadband Communications, 2002.

[15] M. Matariere, K. Baum, and T. P. Krauss, "Cyclic Prefix length Analysis for 4G OFDM systems," IEEE VTC, pp. 543-547, 2004.

[16] M. Tonello, F. Versolatto, B. Bejar, and S. Zazo, "A fitting algorithm for random modeling the PLC channel." IEEE Transactions on Power Delivery, vol. 27(3), pp. 1477-1484, 2012.

[17] R. Hashmat, P. Pagani, and T. Chonavel, "Analysis and modeling of background noise for inhome MIMO-PLC channels." IEEE ISPLC, pp. 316-321, 2012. 